\title{
Effects on LOS may not justify cost of remdesivir for severe COVID-19
}

The impact of remdesivir on hospital length of stay (LOS) may not justify the cost of a course in patients with severe COVID-19 infections, according to finding of a study published in PharmacoEconomics - Open.

The chief executive officer of Gilead Sciences announced in June 2020 that the cost of remdesivir would be $\$ 3120$ per 5-day course for the treatment of COVID-19 in US hospitals, based on the understanding that the drug would shorten LOS by 4 days and reduce hospital costs by $\$ 12000$ per patient. This reduction in LOS was extrapolated from the ACT-1 trial which reported that the median time to hospital discharge was shorter with remdesivir than with placebo (median 11 vs 15 days). Approval of remdesivir by the US FDA specifies that it can only be administered in hospitals.

This study investigated the effects of remdesivir on LOS (time to death or hospital discharge) in 1643 adults ( $\geq 18$ years) with severe COVID-19 who were admitted to the New York-Presbyterian Columbia University Irving Medical Center and the Allen community hospital between March and April 2020, and followed up until 10 June 2020. Most patients were Hispanic or Black and had at least one comorbidity.

The LOS was $1-4$ days in $36 \%$ of patients, $5-8$ days in $23 \%$ of patients, and 9 days or over in $41 \%$ of patients. Rates were similar when patients who died during hospitalisation were excluded. Overall, $67 \%$ of patients with a LOS of $1-4$ days and $70 \%$ of patients with a LOS of $5-8$ days were 60 years of age or over.

Median LOS was considerably shorter than in the ACT-1 trial (7 vs 15 days).

"We found that only $41 \%$ of patients could both receive a 5 -day course of remdesivir and have LOS shortened by 4 days or more, whereas $36 \%$ could have their LOS potentially prolonged to complete therapy," said the authors. "Reevaluating remdesivir's pricing, studying shorter remdesivir treatment courses, developing intranasal remdesivir, and implementing programs to facilitate outpatient intravenous remdesivir administration should be considered," they suggested.

Anderson MR, et al. Hospital Length of Stay for Patients with Severe COVID-19: Implications for Remdesivir's Value. PharmacoEconomics-Open : 14 Dec 2020. Available from: URL: https://doi.org/10.1007/s41669-020-00243-6 